\title{
Effect of brachial-ankle pulse wave velocity combined with blood pressure on cardio-cerebrovascular events
}

\author{
YUNTAO WU ${ }^{1,2^{*}}$, YAJING ZHANG ${ }^{2,3^{*}}$, JINGSHENG GAO $^{2}$, SHULI MAN $^{1}$, JIANONG XING $^{4}$, \\ ZHIWEI CAO ${ }^{5}$, SHAOMIN SONG ${ }^{6}$, SHOULING WU ${ }^{2}$ and WENYUAN GAO ${ }^{1}$ \\ ${ }^{1}$ School of Pharmaceutical Science and Technology, Tianjin University, Tianjin 300072; ${ }^{2}$ Department of Cardiology, \\ Kailuan Hospital, North China University of Science and Technology; ${ }^{3}$ Graduate School, \\ North China University of Science and Technology; ${ }^{4}$ Department of Cardiology, The Third People's Hospital; \\ ${ }^{5}$ Department of Cardiology, The People's Hospital of Luanxian; ${ }^{6}$ Department of Cardiology, \\ Linxi Hospital of Kailuan, Tangshan, Hebei 063000, P.R. China
}

Received November 20, 2018; Accepted July 19, 2019

DOI: $10.3892 /$ etm.2019.8149

\begin{abstract}
The aim of the present study was to evaluate the effect of brachial-ankle pulse wave velocity (baPWV) combined with blood pressure (BP) on cardio-cerebrovascular events. Participants who received health examinations during the periods 2010-2011, 2012-2013 and 2014-2015 were recruited. The participants were divided into four groups according to their BP and baPWV levels as follows: Normotension + low baPWV, normotension + high baPWV, hypertension + low baPWV, and hypertension + high baPWV. The cumulative incidence of cardio-cerebrovascular events was calculated using life-table analysis, and the associations of BP and baPWV with cardio-cerebrovascular events were analyzed using a multivariate Cox proportional hazards regression model. Receiver operating characteristic curves were used to calculate the predictive values of baPWV combined with BP, baPWV alone or BP alone for cardio-cerebrovascular events by comparing their area under the curve (AUC) using the normal approximation method. There were 20,310 participants with a mean age of $50.13 \pm 0.09$ years in the present study, including 13,240 males. A total of 278 participants developed a cardio-cerebrovascular event after a mean follow-up period
\end{abstract}

Correspondence to: Professor Wenyuan Gao, School of Pharmaceutical Science and Technology, Tianjin University, 92 Weijin Road, Tianjin 300072, P.R. China

E-mail: 839892070@qq.com

Dr Shouling Wu, Department of Cardiology, Kailuan Hospital, North China University of Science and Technology, 57 Xinhua West Road, Tangshan, Hebei 063000, P.R. China

E-mail: 1831701945@qq.com

${ }^{*}$ Contributed equally

Key words: brachial-ankle pulse wave velocity, blood pressure, cardio-cerebrovascular events, predictive value, Kailuan study of $3.34 \pm 1.82$ years. The cumulative incidence of cardio-cerebrovascular events in the normotension + low baPWV, normotension + high baPWV, hypertension + low baPWV and hypertension + high baPWV groups was $0.2,0.9,0.8$ and $3.1 \%$. Multivariate Cox proportional hazards regression analysis showed that compared with the normotension + low baPWV group, the risks of cardio-cerebrovascular events in the normotension + high baPWV, hypertension + low baPWV and hypertension + high baPWV groups were increased after adjusting for confounding factors, and their hazard ratios (95\% CI) were 4.18 (2.23-7.83), 3.00 (1.39-6.47) and 9.34 (5.14-16.96), respectively. The AUC values for the predictive values of baPWV combined with BP, baPWV alone and BP alone on cardio-cerebrovascular events were calculated to be $0.744,0.677$ and 0.698 , respectively. In conclusion, high baPWV accompanied by hypertension could increase the risk of cardio-cerebrovascular events. The predictive value of baPWV combined with BP on cardio-cerebrovascular events is superior compared with that of either baPWV or BP alone.

\section{Introduction}

Hypertension is a risk factor for cardio-cerebrovascular events, which leads to a series of severe complications, including myocardial infarction (MI), heart failure, stroke and chronic kidney disease (1-4). Atherosclerosis is not only regarded as a sign of vascular aging, but also a risk factor for potential cardio-cerebrovascular events $(5,6)$. Previous research showed that the risk of cardiovascular death exceeds $50 \%$ in hypertensive patients compared with non-hypertensive individuals, even if the blood pressure (BP) of patients is reduced to $140 / 90 \mathrm{mmHg}$ after therapy $(7,8)$. In addition, atherosclerosis serves an important role in the prognosis of cardio-cerebrovascular events (7). Niiranen et al (7) found that arteriosclerosis accompanied with hypertension is a risk factor for cardio-cerebrovascular mortality after a 12.6-year follow-up study of 2,127 cases in the general population. Song et al (9) observed a higher incidence of stroke in patients with arteriosclerosis and hypertension in a 4.5-year follow-up 
study of 3,310 hypertensive patients, especially those with higher BP. Therefore, from these previous observations it could be speculated that the assessment of arteriosclerosis combined with BP maybe an improved predictor for cardio-cerebrovascular events. However, these previous studies on the correlation between cardio-cerebrovascular events and arteriosclerosis combined with BP have certain shortcomings, namely the limited number of cases and specific populations.

The carotid-femoral pulse wave velocity (cfPWV) is considered the gold standard for assessing arteriosclerosis, but its measurement is relatively complex $(10,11)$. The use of cfPWV is often limited in research due to the inconvenience associated with the measuring devices and processes used. The pulse volume waveforms were recorded through a semoconductor pressure sensor, with the sample acquisition frequency for PWV set at 1,200 Hz (12). There is a good correlation between brachial-ankle pulse wave velocity (baPWV) and cfPWV (13). The baPWV is a simple and reproducible measurement that has the potential to be a sensitive tool for evaluating arteriosclerosis. Indeed, a previous study has shown that the correlation between baPWV and cardiovascular risk factors is stronger compared with that of cfPWV (9). Therefere, the aim of the present study was to evaluate the effect of baPWV combined with BP on cardio-cerebrovascular events. The Kailuan study (registration number: ChiCTR-TNC-11001489) is a study assessing the risk factors of cardiovascular diseases based on functional community populations, which consists of five elements: Population, region, system, policy and institution. In order to clarify the prevalence of chronic diseases among the occupational population in China, since June 2006, health check-ups were conducted and data was collected concerning the endpoint events, every 2 years, for employees and retirees of the Kailuan Group. From January 2006 to June 2007, 101510 on-the-job and retired workers took part in health examination, 81,110 were males and 204,00 were females. Thus far, this study has measured and collected baPWV data from parts of the population between 2010 and 2015. Based on data collected from the Kailuan study, the association between baPWV combined with BP and cardio-cerebrovascular events plus possible risk factors was analyzed in the present study.

At present, primary hypertension accounts for the vast majority of cases of hypertension in China (95\%), and is often considered to be the leading risk factor for cardiovascular mortality (14-16). Previous studies have stated that 200 million people in China have hypertension, but few receive treatment or achieve appropriate control of their BP (17-25). Since, primary hypertension is so common, and is highly prevalent in the Kailuan study cohort, primary hypertension was the focus of the present study and patients with secondary hypertension, including aldosteronism, pheochromocytoma and renin-secreting tumor, were excluded.

The prevalence of diabetes mellitus (DM) in China has increased significantly over recent decades, and national representative surveys reveal a rise from $\sim 0.9 \%$ in adults aged $>30$ years in 1980 to $11.6 \%$ in adults aged $>18$ years in 2010 (26,27). Previous studies have reported that the overall combined prevalence of diagnosed and undiagnosed diabetes in China increased from $\sim 2.5$ to $\sim 10.9 \%$ between 1994 and 2013 (28,29). Another study suggested that China has overtaken India and has become the global epicenter of a diabetes epidemic, with $\sim 92.4$ million adults affected (30). As a result of rapid economic development, urbanization and aging, China is facing a high and increasing burden of diabetes (31) and is thought to have had the largest number of individuals with diabetes in the world in 2015, with extrapolation of existing data suggesting that this will continue until at least 2030 (32). Due to the high prevalence of DM and its importance to general health, DM was evaluated as a confounding factor in the present study.

\section{Materials and methods}

Research groups. The study examined four cohorts from the Kailuan cohort study (Shown in the schematic diagram): i) Stroke cohort: According to the sex and age distribution indicated by a $1 \%$ sample of the 2005 national population $\geq 40$ years of age, and considering every 2 years as an age group, personnel at the Stroke Clinical Experimental and Research Center, Capital University of Medical Sciences Affiliated Tiantan Hospital (Beijing, China) selected participants from the 101,510 individuals in the Kailuan study who participated in health examinations between January 1, 2006 and December 31, 2007 using stratified random sampling. The total number of individuals selected by sampling was 5,852 , of whom 5,440 were eligible; among these, 5,219 had completed baPWV tests in the period between January 1, 2011 and December 31, 2012 and were included in the present study. ii) The aged population cohort: Retired participants in the Kailuan study with health examination results who were $>60$ years old between January 1, 2010 and December 31, 2011 are the alternative population. Using a cluster sampling method, the number sampled ( $25 \%$ of the total) was 3,064 , of whom 2,464 were selected as the research cohort. iii) Pregnancy-induced hypertension cohort: From October 1976 to December 2008, 4,676 women, whose average SBP and DBP was $120.60 \pm 16.82 \mathrm{mmHg}$ and $71.82 \pm 11.74 \mathrm{mmHg}$ respectively, with single-child deliveries participated in their first health examinations in 11 hospitals of the Kailuan study between January 1, 2010 and December 31, 2015. iv) Peripheral vascular disease examination cohort: The population who received health examinations in Kailuan General Hospital and Kailuan Qianjiaying Hospital, and agreed to assessment using baPWV measurements between January 1, 2010 and December 31, 2015, of whom 14,424 were selected after baPWV detection. A total of 2464 cases enrolled in Cohort II, but only 1754 were selected based on baPWV detection and 1754 were included in the cohort II. A total of 22,681 individuals underwent baPWV detection; however, 2,096 were omitted due to the lack of BP measurements and 275 were omitted due to a history of cardio-cerebrovascular disease, leaving 20,310 participants in the current study.

Inclusion criteria and exclusion criteria. Inclusion criteria: Individuals in the Kailuan group who received health examinations in 2010, 2012 and 2014, specifically baPWV and BP measurements during 2010-2011, 2012-2013 and 2014-2015. They consented to participate in this study and signed informed consent. Exclusion criteria: i) Lack of baPWV or BP data; ii) individuals suffering from peripheral vascular diseases; iii) serious disability resulting in the inability to receive examinations; and/or iv) lack of consent to participate 
in this study. The present study was conducted in accordance with The Declaration of Helsinki and was approved by the Kailuan General Hospital Ethics Committee.

Data collection. The collection of epidemiological data, anthropometric and laboratory measurements has been described in detail previously (33). The biochemical data and baPWV data were collected concurrently.

baPWV assay. baPWV was measured between 7:00 and 9:00 a.m. using a BP-203RPEIII networked arteriosclerosis test device [Omron Healthcare (China) Co., Ltd] at room temperature. Sex, age, height and weight were also recorded. Smoking was not allowed and a resting period of $>5$ min was required prior to measurements being recorded. Examination was performed on the left and right brachia and ankles with subjects in the supine position. The collection of epidemiological, anthropometric and laboratory data was in accordance with the 2017 ACC/AHA Hypertension Guidelines (34) for the Prevention, Detection, Evaluation, and Management of High Blood Pressure in Adults, as were the specifications of the apparatus for measuring baPWV and BP. The distance between baPWV sampling points was calculated automatically according to the height of the subject. The path length from the suprasternal notch to the ankle (La) was calculated using the following formula: $\mathrm{La}=0.8129 \mathrm{x}$ height $(\mathrm{cm})+12.328$. The path length from the suprasternal notch to the brachium $(\mathrm{Lb})$ was calculated using the following formula: $\mathrm{Lb}=0.2195 \mathrm{x}$ height $(\mathrm{cm})-2.0734$. The baPWV was calculated according to the following formula: baPWV=(La-Lb)/Tba $\mathrm{m} / \mathrm{sec}$ where Tba is the time interval between the front wave of the brachial waveform and the ankle waveform during simultaneous measurements of baPWV on both sides of the body. Because some studies proved the association between baPWV and the atherosclerotic vascular damage and cardiovascular risk, and we think the higher detection values is more likely to reflect the actual situation and may be helpful in the analysis of clinical outcome in the study. The highest readings were chosen as the representative values for each individual. According to the 2017 ACC/AHA Guideline for the Prevention, Detection, Evaluation, and Management of High Blood Pressure in Adults: baPWV $<1,400 \mathrm{~cm} / \mathrm{sec}$ was regarded as normal arterial stiffness and baPWV $\geq 1,400 \mathrm{~cm} / \mathrm{sec}$ as atherosclerosis and Ankle brachial index $\leq 0.9$ was defined as peripheral vascular disease (34).

BP measurements. BP was measured between 7:00 and 9:00 a.m. BP was measured using calibrated mercury sphygmometers in the right brachial artery after $15 \mathrm{~min}$ of rest. The first and the fifth Korotkoff sounds were recorded as systolic BP (SBP) and diastolic BP (DBP), respectively (35). These two Korotkoff sounds were continuously measured three times, from which the average was calculated. Smoking was defined as having smoked $>1$ cigarette/day over the last 12 months, drinking was defined as having consumed $100 \mathrm{ml}$ strong spirit (alcohol content $>50 \%$ ) daily for $\geq 1$ year and physical exercise was defined as performing $\geq 3$ exercise sessions/week with each lasting $\geq 30 \mathrm{~min}$.

Follow-up time and definition of endpoints. The start point was defined as the time of baPWV data collection from January 1, 2010 and the last follow-up time was December 31,
2015. Cardio-cerebrovascular events were defined as an endpoint, which mainly comprised stroke (hemorrhagic and ischemic) and MI. If the events occurred more than twice in a year, the first cardio-cerebrovascular event was considered as the endpoint. The endpoints were carefully recorded by experienced professionals, and all diagnoses were confirmed by professional physicians according to medical records. Throughout the research process, all patients/subjects were either past or present employees of Kailuan Co., Ltd., and no records of patients/subjects were lost during the follow-up.

Associated definitions. Hypertension was defined as SBP $\geq 140 \mathrm{mmHg}$ and/or DBP $\geq 90 \mathrm{mmHg}$, with a history of hypertension or currently taking antihypertensive medication even with SBP $<140 \mathrm{mmHg}$ and DBP $<90 \mathrm{mmHg}$ (36). Normotension was defined as BP $\leq 140 / 90 \mathrm{mmHg}$ without a history of hypertension or antihypertensive treatment; high baPWV was defined as $\geq 1,400 \mathrm{~cm} / \mathrm{sec}$ whereas low baPWV was defined as $<1,400 \mathrm{~cm} / \mathrm{sec}$. Diabetes was defined as fasting blood glucose $(\mathrm{FBG}) \geq 7.0 \mathrm{mmol} / \mathrm{l}$, with a history of diabetes or currently taking hypoglycemic medication even with FBG $<7.0 \mathrm{mmol} / \mathrm{l}$. (37).

Statistical analysis. The Wald test was performed as part of the Cox hazard model. All data were analyzed using SPSS 13.0 statistical software (SPSS, Inc.). Normally distributed data were presented as mean \pm standard error of the mean. Student's t-test was used for comparisons between two groups, whereas one-way ANOVA was applied for comparisons among $>2$ independent groups. Equal variance was compared using the least significant difference or Dunnett's T3 post hoc test. Any measured data that differed from a normal distribution were log-transformed to normal distribution, and the corresponding parameter ANOVA test was applied in the results. All counted data were presented as $\mathrm{n}(\%)$, where the $\chi^{2}$ test was used for comparisons between groups. The objects were divided into different groups according to baPWV and BP. The cumulative incidence of endpoint events was calculated using the life table method. Any differences were compared using the log-rank test, and hazard ratios (HR) and 95\% CI values for the different groups of cardio-cerebrovascular events were calculated using the Cox proportional hazards model. Wald test was performed as part of the Cox hazard model The predictive value of combined measurements of baPWV and BP, baPWV alone and BP alone on cardio-cerebrovascular events was assessed using receiver operating characteristic (ROC) curves (38). The normal approximation method was used to evaluate the area under the ROC curve (AUC). The larger the AUC, the more accurate the prediction of endpoint events. Sensitivity analysis: In order to exclude the effects of antihypertensive, hypoglycemic and lipid-lowering medication in the research, statistical analyzes were also reconducted following the exclusion of the population undergoing drug treatments. $\mathrm{P}<0.05$ was considered to indicate a statistically significant difference.

\section{Results}

Participant characteristics. In the study, there were 20,310 participants (13,240 male and 7,070 female) with an average age of $50.13 \pm 0.09$ years. According to the values of baPWV and 


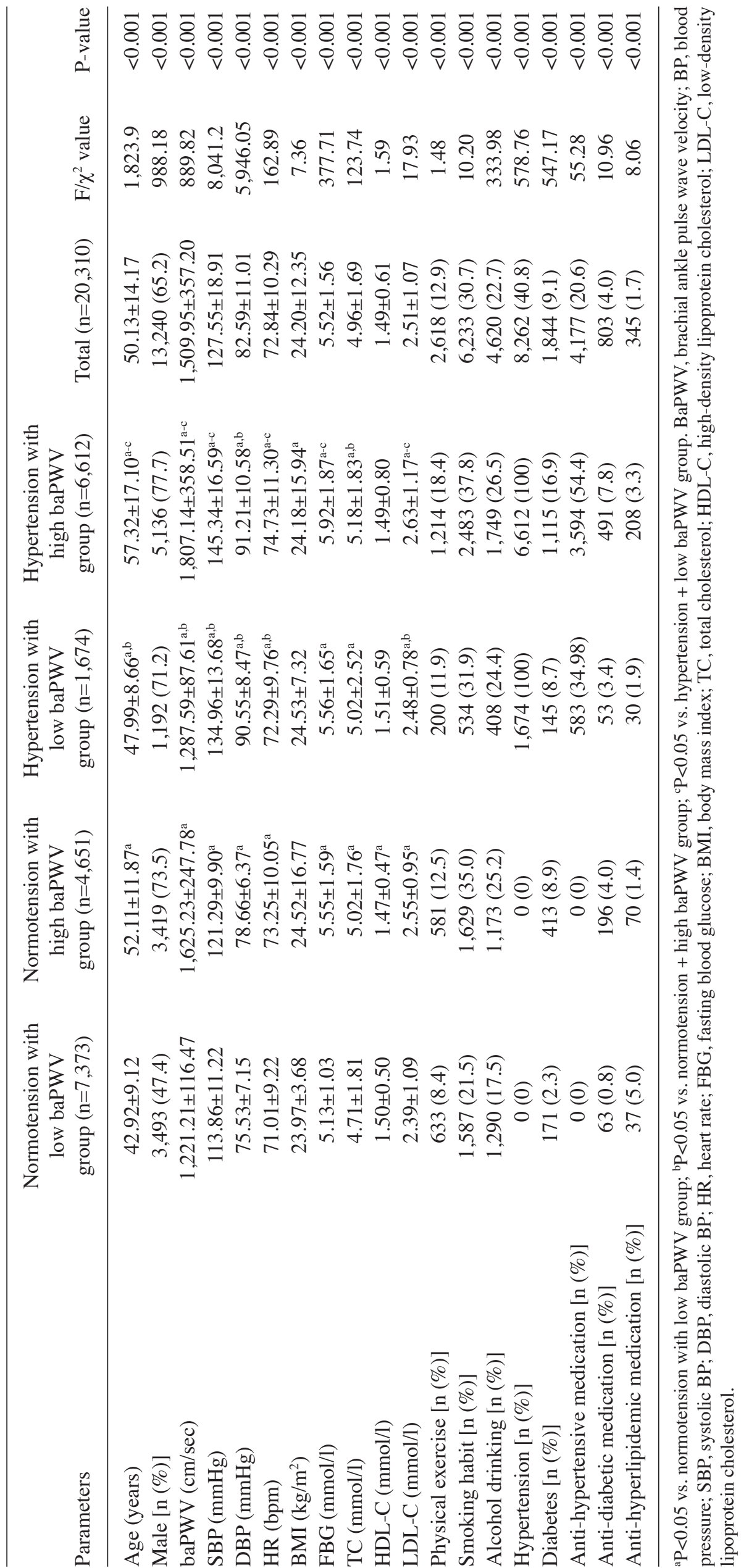


Table II. Cumulative incidence of cardio-cerebrovascular events in different groups.

\begin{tabular}{|c|c|c|c|c|c|c|}
\hline \multirow[b]{2}{*}{$\begin{array}{l}\text { Cardio-cerebrovascular } \\
\text { events }\end{array}$} & \multicolumn{4}{|c|}{ Groups } & \multirow[b]{2}{*}{ Total } & \multirow[b]{2}{*}{ P-value } \\
\hline & $\begin{array}{l}\text { Normotension }+ \\
\text { low baPWV }\end{array}$ & $\begin{array}{l}\text { Normotension }+ \\
\text { high baPWV }\end{array}$ & $\begin{array}{c}\text { Hypertension + } \\
\text { low baPWV }\end{array}$ & $\begin{array}{l}\text { Hypertension + } \\
\text { high baPWV }\end{array}$ & & \\
\hline Myocardial infarction, [n (\%)] & $4(0.1)$ & $12(0.3)$ & $7(0.4)$ & $52(0.8)$ & $75(0.4)$ & $<0.001$ \\
\hline Stroke $[\mathrm{n}(\%)]$ & $9(0.1)$ & $31(0.7)$ & $7(0.4)$ & $158(2.4)$ & $205(1.0)$ & $<0.001$ \\
\hline Ischemic stroke & $8(0.1)$ & $27(0.6)$ & $7(0.4)$ & $142(2.1)$ & $184(0.9)$ & $<0.001$ \\
\hline Hemorrhagic stroke & $1(0.01)$ & $5(0.1)$ & $2(0.1)$ & $17(0.3)$ & $25(0.1)$ & $<0.001$ \\
\hline Total $[\mathrm{n}(\%)]$ & $13(0.2)$ & $43(0.9)$ & $14(0.8)$ & $208(3.1)$ & $278(1.4)$ & $<0.001$ \\
\hline
\end{tabular}

Results demonstrate a statistically significant difference in the incidence of cardio-cerebrovascular events between any two groups among all 4 research groups $(\mathrm{P}<0.001)$. baPWV, brachial-ankle pulse wave velocity. Among the 278 cases, 2 cases experienced both MI and stroke, and 4 cases experienced both ischemic stroke and hemorrhagic stroke.

BP, participants were divided into four groups: Normotension + low baPWV ( $=7,373)$; normotension + high baPWV $(n=4,651)$; hypertension + low baPWV $(n=1,674)$; and hypertension + high baPWV $(n=6,612)$. Compared with other groups, participants from the hypertension + high baPWV group exhibited significantly higher age, male ratio, baPWV, SBP, DBP, FBG, heart rate, total cholesterol, low density lipoprotein cholesterol, physical exercise, DM, and use of antihypertensive drugs, hypoglycemic drugs and lipid-lowering drugs (Table I).

Incidence of cardio-cerebrovascular events between the four groups. Within the follow-up period of 3.34 \pm 1.82 years, there were 278 cases of cardio-cerebrovascular events. These included 75 cases of MI and 205 cases of stroke (two cases overlapped). Among the 205 cases of stroke, there were 184 cases of ischemic stroke and 25 cases of hemorrhagic stroke (four cases overlapped). Compared with the normotension + low baPWV group, the other three groups displayed significantly increased cumulative incidences of cardio-cerebrovascular events, MI, stroke, ischemic stroke and hemorrhagic stroke. In particular, the cumulative incidences exhibited by the hypertension + high baPWV group $(3.1,0.8,2.4,2.1$ and $0.3 \%$ for cardio-cerebrovascular events, $\mathrm{MI}$, stroke, ischemic stroke and hemorrhagic stroke, respectively), were significantly higher compared with those in the normotension + low baPWV group $(0.2,0.1,0.1,0.1$ and $0.01 \%$, respectively). Using the log-rank test, the cumulative incidences of the end-point events demonstrated by the different groups were revealed to be statistically significant (Table II).

Endpoint events calculation using Cox proportional hazard model. For the present study, cardio-cerebrovascular events, MI, stroke, ischemic stroke and hemorrhagic stroke were defined as dependent variables whereas the different four experimental groups were defined as independent variables, and the normotension + low baPWV group was used as a control. Cox proportional hazard model analysis was then performed after adjusting for confounding factors, including sex, age, smoking, drinking, physical exercise, TC and the use of antihypertensive medication, hypoglycemic medication or lipid-lowering medication. Compared with control group, the
HR values (95\% CI) of cardio-cerebrovascular events in the normotensive + high baPWV, hypertension + low baPWV and hypertension + high baPWV groups were 4.18 (2.23-7.83), 3.00 (1.39-6.47) and 9.34 (5.14-16.96), respectively; the HR values $(95 \% \mathrm{CI})$ of MI were 3.11 (0.98-9.87), 4.60 (1.31-16.18) and 7.14 (2.42-21.04), respectively; the HR values $(95 \% \mathrm{CI})$ of stroke were 4.65 (2.20-9.83), 2.20 (0.81-5.99) and 10.37 (5.07-21.21), respectively; the HR values (95\% CI) of ischemic stroke were 4.66 (2.10-10.34), 2.55 (0.91-7.15) and 10.83 (5.07-23.12), respectively; and the HR values (95\% CI) of hemorrhagic stroke were 5.93 (0.67-52.78), 3.62 (0.31-43.03) and 6.51 (0.74-57.74), respectively (Model III; Table III). In addition, different groups were established using values from the baPWV and BP measurements. These were used as independent variables, whereas cardio-cerebrovascular events, MI, stroke, ischemic stroke and hemorrhagic stroke were defined as dependent variables. After adjusting for sex, age, smoking, drinking, physical exercise, TC, taking antihypertensive medication, hypoglycemic medication and lipid-lowering medication. The results showed that the risk of cardio-cerebrovascular events exhibited positive associations with increases in baPWV or BP (data not shown).

Predictive values of cardio-cerebrovascular events using baPWV combined with BP. ROC curves were plotted of baPWV combined with BP, baPWV alone and BP alone for the prediction of cardio-cerebrovascular events, MI, stroke, ischemic stroke and hemorrhagic stroke. For baPWV combined with BP, baPWV alone and BP alone, respectively, the AUC values were calculated to be $0.744,0.677$ and 0.698 for the predication of cardio-cerebrovascular events (Fig. 1A); $0.726,0.650$ and 0.690 , respectively, for the prediction of MI (Fig. 1B); 0.750, 0.686 and 0.700 , respectively, for the prediction of stroke (Fig. 1C); 0.750, 0.684 and 0.703, respectively for prediction of ischemic stroke (Fig. 1D), and 0.722 , 0.658 and 0.676 , respectively for the prediction of hemorrhagic stroke (Fig. 1E). The AUC values were tested further using the normal approximation method and were calculated to be statistically significant $(\mathrm{P}<0.05)$, and the AUC of baPWV combined with BP was maximal, and it could be applied to the prediction of all 5 diseases categories. 


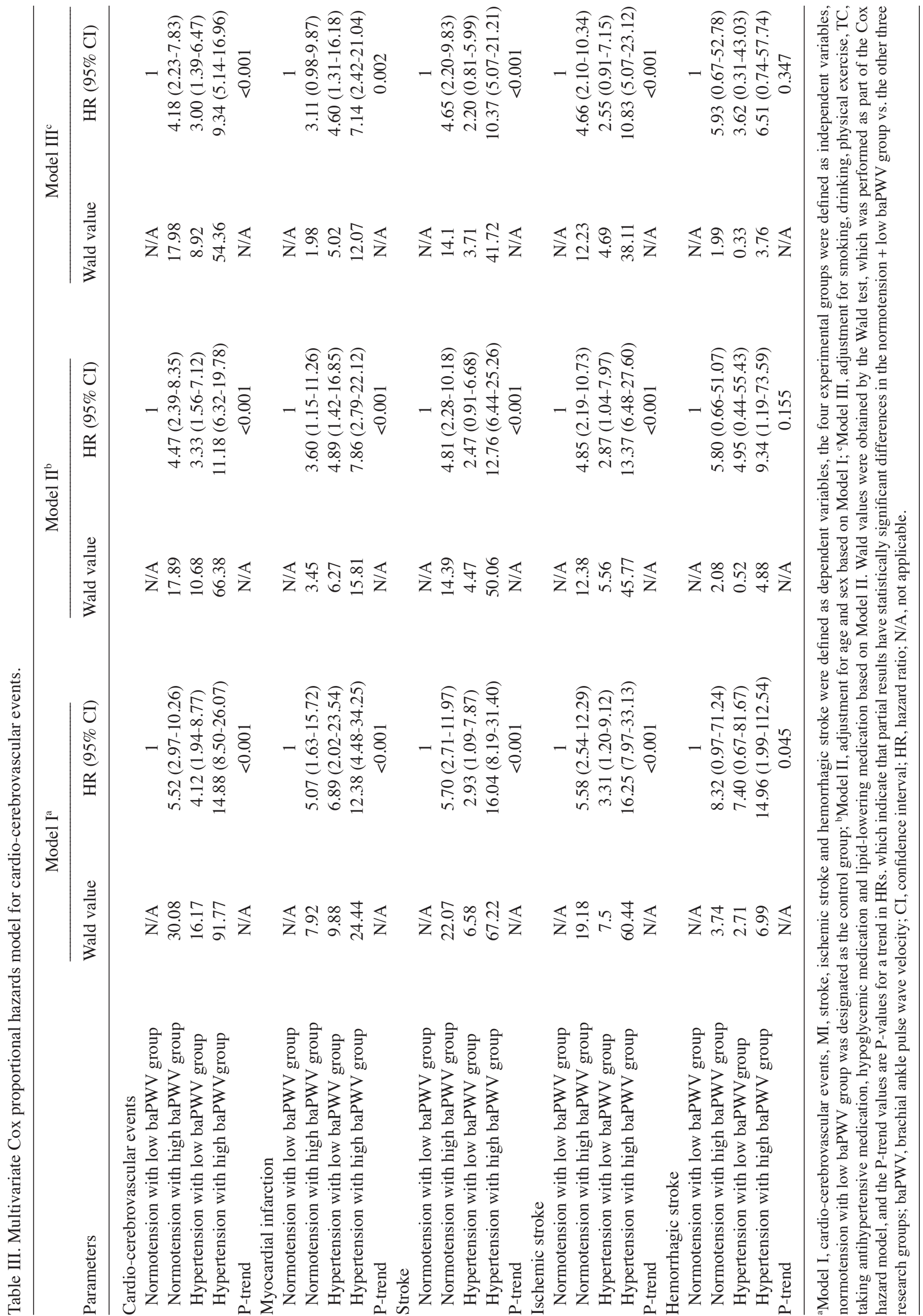


A

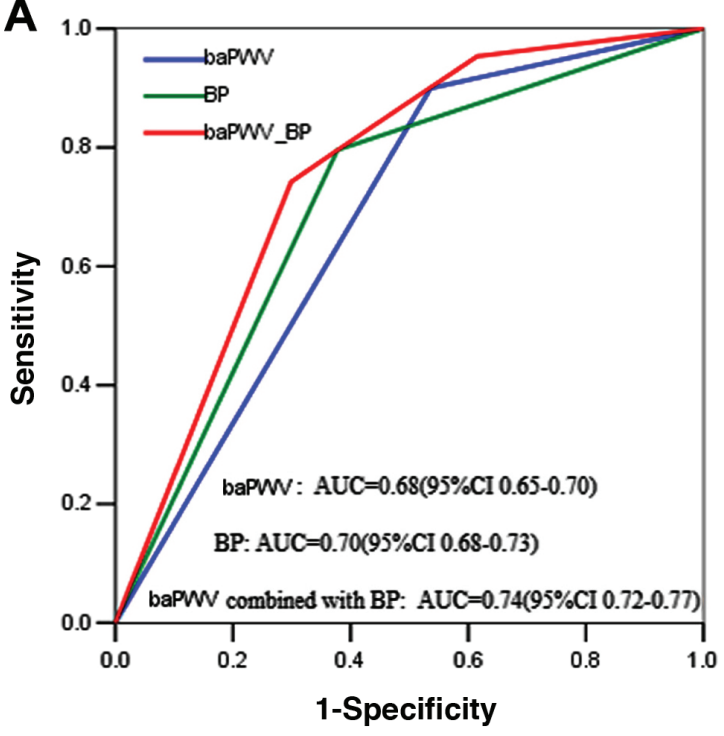

C

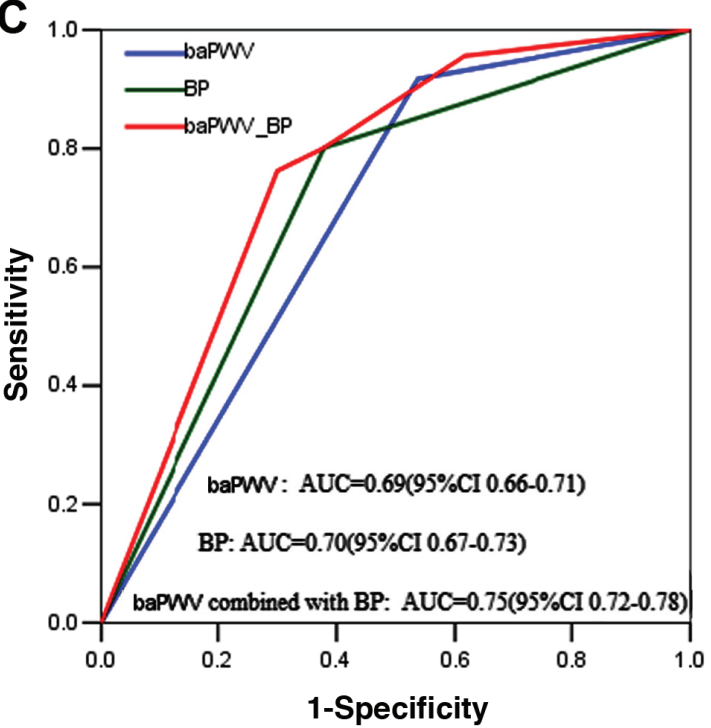

B

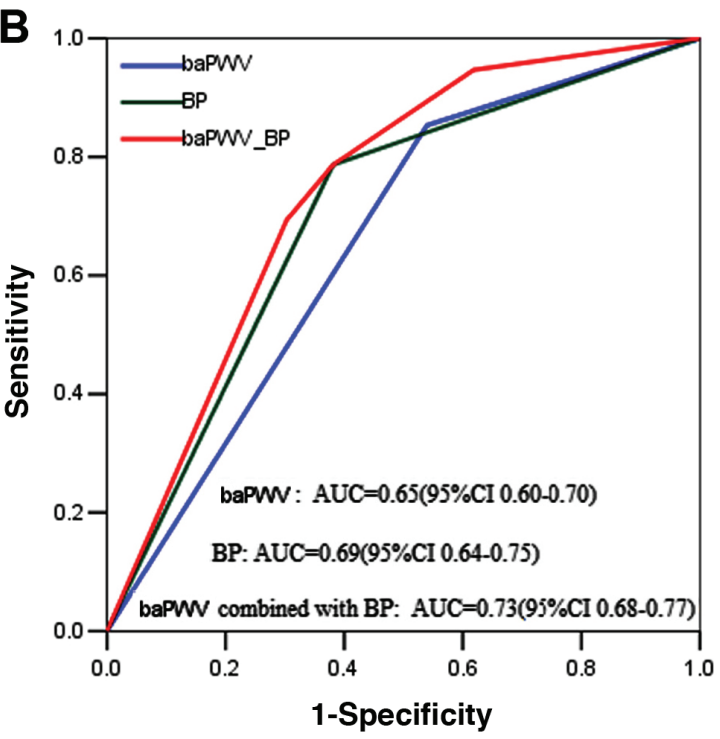

D

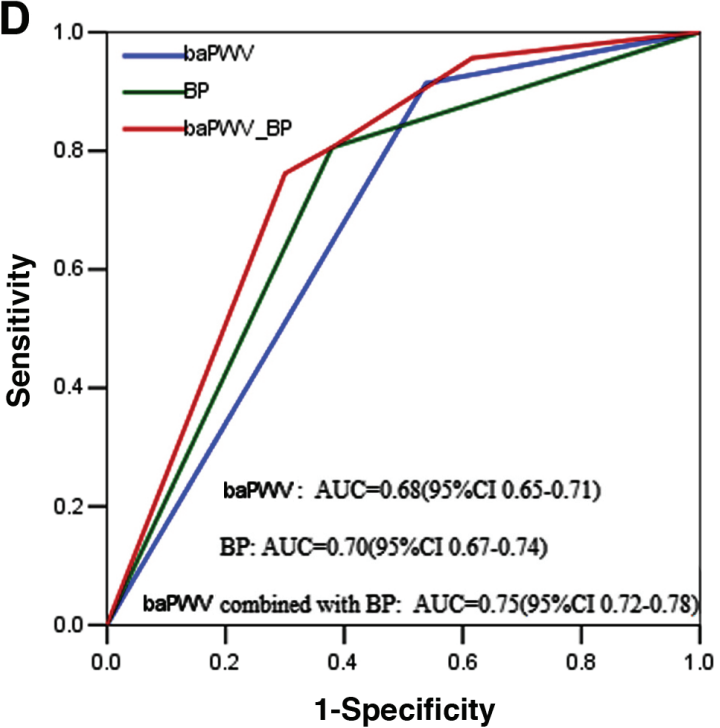

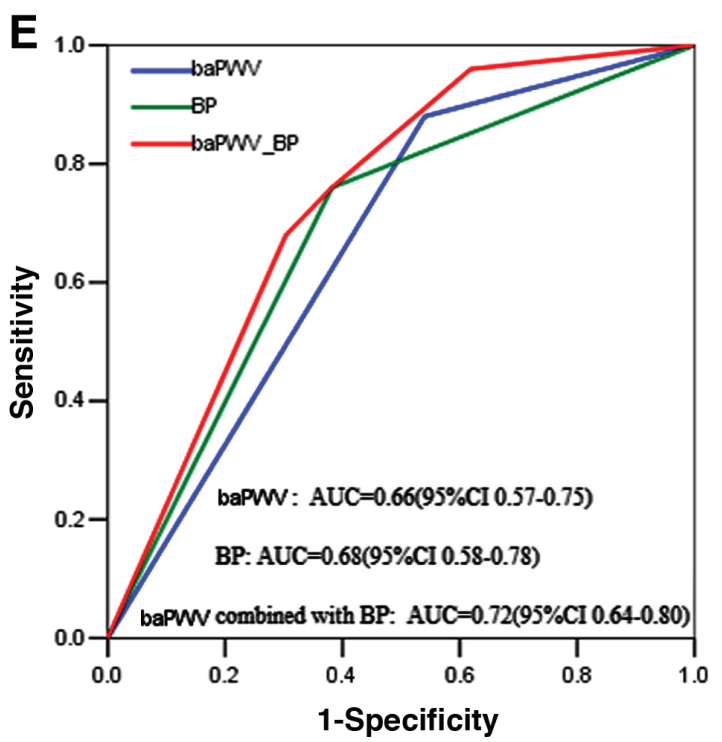

Figure 1. Receiver operating characteristic curve for predicting (A) cardio-cerebrovascular events, (B) myocardial infarction, (C) stroke, (D) ischemic stroke and (E) hemorrhagic stroke based on baPWV alone, BP alone and baPWV combined with BP. baPWV, brachial-ankle pulse wave velocity; BP, blood pressure; AUC, area under the curve; CI, confidence interval. 


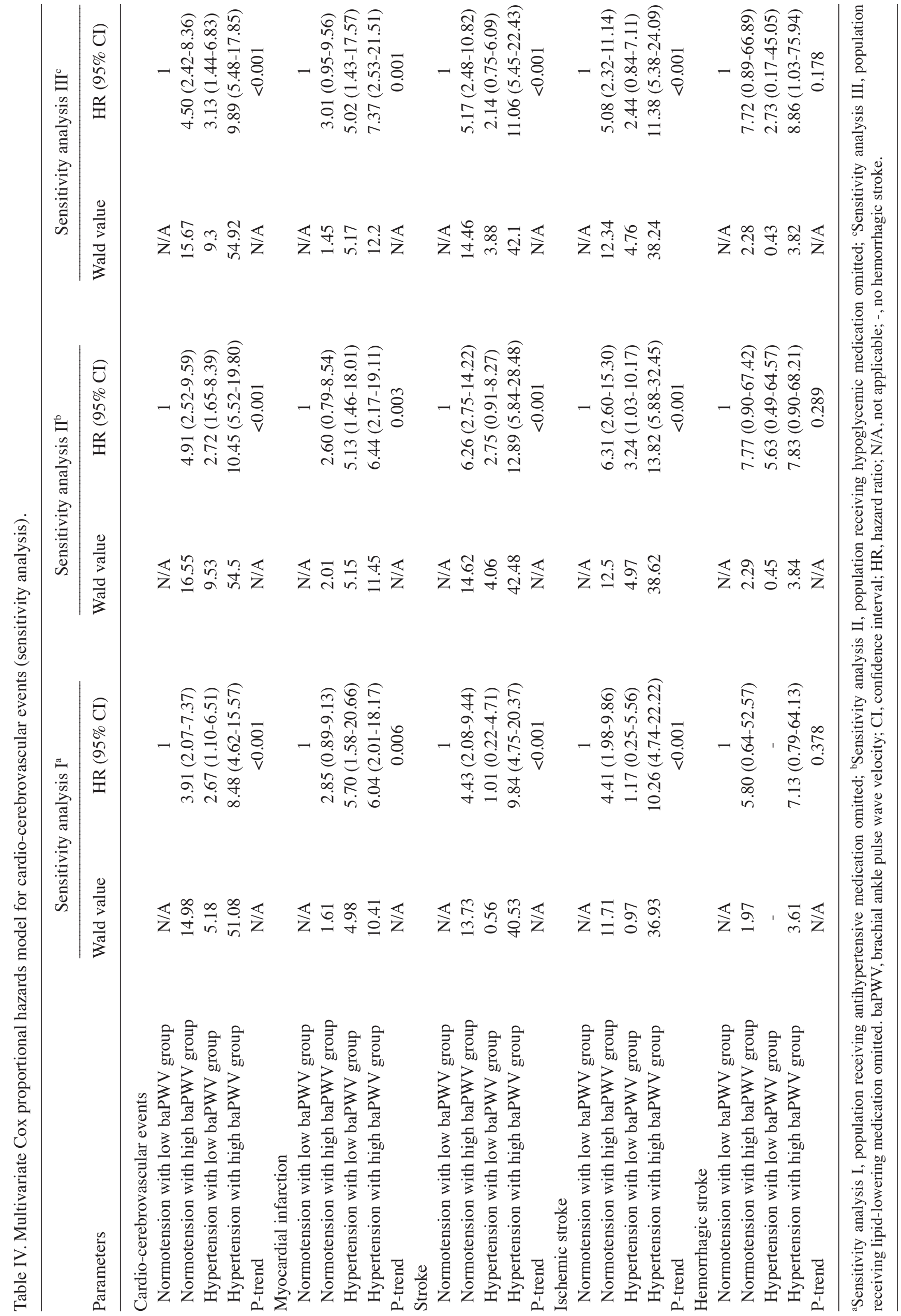


Sensitivity analysis. Cox regression analysis was performed after the exclusion of participants currently receiving antihypertensive medication, hypoglycemic medication or lipid-lowering medication. Following the exclusion of participants receiving antihypertensive medication, compared with the normotension + low baPWV group, the HR values (95\% CI) of cardio-cerebrovascular events, MI, stroke, ischemic stroke and hemorrhagic stroke were 8.48 (4.62-15.57), 6.04 (2.01-18.17), 9.84 (4.75-20.37), 10.26 (4.74-22.22) and 7.13 (0.79-64.13), respectively, for the hypertension + high baPWV group (sensitivity analysis I; Table IV). Following the exclusion of participants receiving hypoglycemic medication, the HR values (95\% CI) were 10.45 (5.52-19.80), 6.44 (2.17-19.11), 12.89 (5.84-28.48), $13.82(5.88-32.45)$ and 7.83 (0.90-68.21) for cardio-cerebrovascular events, MI, stroke, ischemic stroke and hemorrhagic stroke, respectively, for the hypertension + high baPWV group (sensitivity analysis II; Table IV). After omitting the participants receiving lipid-lowering medication, the HR values (95\% CI) were 9.89 (5.48-17.85), 7.37 (2.53-21.51), 11.06 (5.45-22.43), 11.38 (5.38-24.09) and 8.86 (1.03-75.94) for cardio-cerebrovascular events, MI, stroke, ischemic stroke and hemorrhagic stroke, respectively, for the hypertension + high baPWV group (sensitivity analysis III; Table IV). These statistical analyses, indicate that high baPWV combined with high $\mathrm{BP}$ is a risk factor for cardio-cerebrovascular events.

\section{Discussion}

The results of the present study indicate that the cumulative incidences of cardio-cerebrovascular events, MI and stroke in normotensive patients with high baPWV, and hypertensive patients with low or high baPWV were higher compared with that in normotensive patients with low baPWV. The cumulative incidences of the aforementioned diseases in the hypertension + high baPWV group were markedly higher compared with those in the normotension + low baPWV group. In addition, the cumulative incidence of cardio-cerebrovascular events in the normotension + high baPWV group was higher compared with that in the hypertension + low baPWV group. Niiranen et al (7) observed that after a 12.6-year follow-up of 2,127 subjects, the cumulative incidence of cardiovascular events was 1.9, 8.1, 14.9 and $20.0 \%$ in normotension with low cfPWV, normotension with high cfPWV, hypertension with low cfPWV group and hypertension with high cfPWV groups, respectively.

The incidence of cardio-cerebrovascular events, MI and stroke were not only increased in the high baPWV groups. Following adjustments for confounding factors, including age and sex, the risks of the occurrence of cardio-cerebrovascular events, MI and stroke in the normotension + high baPWV, hypertension + low baPWV and hypertension + high baPWV groups were all increased compared with those in the normotension + low baPWV group. These findings showed that in the hypertension + high baPWV group compared with the normotension + low baPWV group, the occurrence risk increased 9.34-fold for cardio-cerebrovascular events, 7.14-fold for MI and 10.37-fold for stroke. However, Song et al (9) found that compared with baPWV in the lowest quartile with adequate hypertension control, the occurrence risk increased by only 2.57-fold with baPWV in the highest quartile with inadequate hypertension control, which may be due to the increased focus on the population receiving antihypertension medication, leading to reduction in the risk of stroke. The present study also indicated that high baPWV maybe a risk factor for stroke, but the risk of stroke in the hypertension + low baPWV group was not markedly greater compared with that in the normotension + low baPWV group, suggesting that baPWV is a superior predictor of stroke compared with BP. The study confirmed the findings of previous studies that high baPWV and hypertension are risk factors for cardio-cerebrovascular events (39-42), and also revealed that high baPWV combined with hypertension has a superior predictive value for cardio-cerebrovascular events compared with only high baPWV alone or hypertension alone. This suggests that when evaluating the risk of cardio-cerebrovascular inpatients, baPWV should be considered as an important assessment parameter target besides BP and arterial stiffness.

When the patients currently receiving antihypertensive medication were excluded, it was found that in the hypertension + high baPWV group the risk of cardio-cerebrovascular events, MI and stroke were significantly increased compared with those in the other three groups but the HR values were reduced compared with the group treated with antihypertensive medication. This suggests that the hypertensive patients currently on antihypertensive medications are at a high-risk for cardio-cerebrovascular events. Walsh et al (43) found that treatment of hypertension was an important risk factor for cerebral hemorrhage. Similar patterns of results were observed among the four groups, despite the exclusion of patients taking hypoglycemic or lipid-lowering medication, suggesting that the effect of baPWV combined with BP on cardio-cerebrovascular events is independent of concomitant antihypertensive, hypoglycemic or lipid-lowering medication treatment.

High baPWV accompanied by hypertension increases the risk of cardio-cerebrovascular events caused by arteriosclerosis, suggesting that baPWV may also be an indicator of atherosclerosis. Generally, atherosclerosis is an important risk factor for hypertension, and is caused by vascular wall hardening and blood vessel lumen narrowing (44). Prolonged high BP increases the arterial hemodynamic load, leading to a decrease in arterial compliance and finally to arteriosclerosis $(45,46)$. High baPWV causes damage to targeted organs and induces BP elevation, which reinforce each other through a feedback loop and thereby raise the risk of cardio-cerebrovascular events (7). In addition, the present study indicated that the association of baPWV with stroke exceeds that of BP, potentially due to the systemic effects of atherosclerosis. A cross-sectional study in China demonstrated that $>60 \%$ stroke patients suffer from atherosclerosis (47). Cerebral infarction readily occurs in patients with atherosclerosis due to the presence of cerebral arterial stenosis, in which the affected blood vessels become vulnerable to rupture and bleeding due to stiffness and fragility.

The collection of baPWV measurements is a relatively simple procedure that can be conducted in an ordinary clinical setting, and may be combined with BP monitoring. Detecting baPWV contributes to the early detection of atherosclerosis and prediction of the occurrence of cardio-cerebrovascular events, and the observation of changes in baPWV may also help to determine the effect of antihypertensive therapy. One 
of the most important aims of the antihypertensive recommendations of the European Society of Hypertension is to alleviate atherosclerosis, with reductions of baPWV and BP being equally important (48). Reducing baPWV induces improvements in vessel walls and tissues (49), which are key to successful antihypertensive treatment. Therefore, baPWV measurements have important clinical significance in the evaluation of arterial function in hypertensive patients, monitoring of treatment outcome and assessment of prognosis.

The risk factors associated with cardio-cerebrovascular events were evaluated in the present study, and an analysis of the association of baPWV with hypertension and cardio-cerebrovascular events was conducted. However, risk factors associated with cardio-cerebrovascular events potentially include many diseases and conditions. Special attention should be paid to factors not considered in this study, including controlled diabetes, dyslipidemia and body mass index (BMI). In particular, a previous study demonstrated that BMI values were significantly associated with the occurrence of cardio-cerebrovascular events $(\mathrm{P}=0.020)$ after controlling for multiple confounding factors (50). Although $\mathrm{BMI}$ has been found to be positively associated with BP, the correlation between BMI and BP was substantially poorer in patients taking antihypertensive medication compared with those who were untreated, demonstrating the effectiveness of treatment (51). In other studies, multiple logistic regression analysis revealed that dyslipidemia was significantly associated with age, being male, BMI, FBG and cigarette smoking, suggesting that dyslipidemia is an important risk factor for cardio-cerebrovascular events (52), and multivariable Cox's proportional hazards regression model showed that hyperlipidemia with type II DM increased the risk of cardio-cerebrovascular events (53). As mentioned above, by performing a regression analysis incorporating additional confounding factors, including underlying diseases such as controlled diabetes and dyslipidemia, sex and BMI in further research, the correlation analysis of the present study could potentially be strengthened.

Although thepresentstudy found thathighbaPWV combined with hypertension increased the risks of cardio-cerebrovascular events, MI and stroke, it has a number of limitations. cfPWV, which is recommended by the American Heart Association as the gold standard in the evaluation of atherosclerosis (11), was not measured and compared with baPWV in the present study. However, baPWV has been shown to correlate well with cfPWV, and a number of studies have demonstrated baPWV to be more relevant to cardiovascular risk factors compared with cfPWV (11). Ohkuma et al (54) suggested that baPWV has potential as a new marker of cardiovascular risk over conventional markers, it's easy to obtain and serves as an indicator of atherosclerotic vascular damage. The number of endpoint events is relatively small and the average follow-up time was 3.34 years, which was not sufficient to enable the collection of a large number of endpoint events. However, the large sample size $(n=20,310)$ remedies this shortcoming to a certain degree, and it does not affect the conclusions of the present study. As many confounding factors as possible were considered when evaluating the influence of BP and baPWV on endpoint events, but other confounding factors such as environmental influences were not accounted for. Finally, the research subjects in the present study are a Northern Chinese population, which is not representative of the global population. Therefore, the findings of the present study require further validation in other populations.

In conclusion, the findings presented in the present study suggest that high baPWV combined with hypertension increases the risk of cardio-cerebrovascular events, and the predictive value of high baPWV combined with high BP on cardio-cerebrovascular events is superior compared with either high baPWV or hypertension alone.

\section{Acknowledgements}

Not applicable.

\section{Funding}

No funding was received.

\section{Availability of data and materials}

The datasets used and/or analyzed during the current study are available from the corresponding author on reasonable request.

\section{Authors' contributions}

YW, YZ and WG conceived and designed the study. YW, YZ and JG wrote the manuscript. All data collection and statistical analysis were performed by SM, JX, ZC and SS. SW and WG reviewed and edited the manuscript. JG and SW collected and analysed clinical data. All authors read and approved the final version of the manuscript.

\section{Ethics approval and consent to participate}

The present study was conducted in accordance with The Declaration of Helsinki and was approved by the Kailuan General Hospital Ethics Committee. All participants signed informed consent for participation in this study.

\section{Patient consent for publication}

Not applicable.

\section{Competing interests}

The authors declare that they have no competing interests.

\section{References}

1. Rapsomaniki E, Timmis A, George J, Pujades-Rodriguez M, Shah AD, Denaxas S, White IR, Caulfield MJ, Deanfield JE, Smeeth L, et al: Blood pressure and incidence of twelve cardiovascular diseases: Lifetime risks, healthy life-years lost, and age-specific associations in 1.25 million people. Lancet 383 : 1899-1911, 2014.

2. Muntner P, Davis BR, Cushman WC, Bangalore S, Calhoun DA, Pressel SL, Black HR, Kostis JB, Probstfield JL, Whelton PK, et al: Treatment-resistant hypertension and the incidence of cardiovascular disease and end-stage renal disease: Results from the Antihypertensive and Lipid-Lowering Treatment to Prevent Heart Attack Trial (ALLHAT). Hypertension 64: 1012-1021, 2014. 
3. O'Donnell MJ,Xavier D,Liu L,Zhang H,Chin SL, Rao-MelaciniP, Rangarajan S, Islam S, Pais P, McQueen MJ, et al: Risk factors for ischaemic and intracerebral haemorrhagic stroke in 22 countries (the INTERSTROKE study): A case-control study. Lancet 376 : $112-123,2010$

4. Triantafyllou A, Anyfanti P, Zabulis X, Gavriilaki E, Karamaounas P, Gkaliagkousi E, Petidis K, Pyrpasopoulou A Girasis C, Aslanidis S and Douma S: Accumulation of microvascular target organ damage in newly diagnosed hypertensive patients. J Am Soc Hypertens 8: 542-549, 2014.

5. Xu Y, Wu Y, Li J, Ma W, Guo X, Luo Y and Hu D: The predictive value of brachial-ankle pulse wave velocity in coronary atherosclerosis and peripheral artery diseases in urban Chinese patients. Hypertens Res 31: 1079-1085, 2008.

6. Laurent S, Boutouyrie P, Asmar R, Gautier I, Laloux B, Guize L, Ducimetiere $\mathrm{P}$ and Benetos A: Aortic stiffness is an independent predictor of all-cause and cardiovascular mortality in hypertensive patients. Hypertension 37: 1236-1241, 2001

7. Niiranen TJ, Kalesan B, Hamburg NM, Benjamin EJ, Mitchell GF and Vasan RS: Relative contributions of arterial stiffness and hypertension to cardiovascular disease: The framingham heart study. J Am Heart Assoc 5: pii: e004271, 2016.

8. Blacher J, Evans A, Arveiler D, Amouyel P, Ferrières J, Bingham A, Yarnell J, Haas B, Montaye M, Ruidavets JB, et al: Residual cardiovascular risk in treated hypertension and hyperlipidaemia: The PRIME study. J Hum Hypertens 24: 19-26, 2010.

9. Song Y, Xu B, Xu R, Tung R, Frank E, Tromble W, Fu T, Zhang W, Yu T, Zhang C, et al: Independent and joint effect of brachial-ankle pulse wave velocity and blood pressure control on incident stroke in hypertensive adults. Hypertension 68: 46-53, 2016.

10. Mancia G, De Backer G, Dominiczak A, Cifkova R, Fagard R, Germano G, Grassi G, Heagerty AM, Kjeldsen SE, Laurent S, et al: 2007 Guidelines for the management of arterial hypertension: The task force for the management of arterial hypertension of the European society of hypertension (ESH) and of the European society of cardiology (ESC). J Hypertens 25: $1105-1187,2007$.

11. Townsend RR, Wilkinson IB, Schiffrin EL, Avolio AP, Chirinos JA, Cockcroft JR, Heffernan KS, Lakatta EG, McEniery CM, Mitchell GF, et al: Recommendations for improving and standardizing vascular research on arterial stiffness: A scientific statement from the American heart association. Hypertension 66: 698-722, 2015.

12. Yamashina A, Tomiyama $\mathrm{H}$, Arai $\mathrm{T}$, Hirose $\mathrm{K}$, Koji $\mathrm{Y}$, Hirayama Y, Yamamoto Y and Hori S: Brachial-ankle pulse wave velocity as a marker of atherosclerotic vascular damage and cardiovascular risk. Hypertens Res 26: 615-622, 2003

13. Tanaka H, Munakata M, Kawano Y, Ohishi M, Shoji T, Sugawara J, Tomiyama H, Yamashina A, Yasuda H, Sawayama T and Ozawa T: Comparison between carotid-femoral and brachial-ankle pulse wave velocity as measures of arterial stiffness. J Hypertens 27: 2022-2027, 2009.

14. Ezzati M, Lopez AD, Rodgers A, Vander Hoorn S and Murray CJ; Comparative Risk Assessment Collaborating Group: Selected major risk factors and global and regional burden of disease. Lancet 360: 1347-1360, 2002.

15. He J, Gu D, Chen J, Wu X, Kelly TN, Huang JF, Chen JC, Chen CS, Bazzano LA, Reynolds K, et al: Premature deaths attributable to blood pressure in China: A prospective cohort study. Lancet 374: 1765-1772, 2009.

16. Fang L, Song J, Ma Z, Zhang L, Jing C and Chen D: Prevalence and characteristics of hypertension in mainland Chinese adults over decades: A systematic review. J Hum Hypertens 28: 649-656, 2014.

17. Lewington S, Lacey B, Clarke R, Guo Y, Kong XL, Yang L, Chen Y, Bian Z, Chen J, Meng J, et al: The burden of hypertension and associated risk for cardiovascular mortality in China. JAMA Intern Med 176: 524-532, 2016.

18. Wang JG: Chinese hypertension guidelines. Pulse (Basel) 3: 14-20, 2015.

19. Guan X, Liang H, Xue Y and Shi L: An analysis of China's national essential medicines policy. J Public Health Policy 32: 305-319, 2011

20. Feng XL, Pang $M$ and Beard J: Health system strengthening and hypertension awareness, treatment and control: Data from the China health and retirement longitudinal study. Bull World Health Organ 92: 29-41, 2014.

21. Li Y, Yang L, Wang L, Zhang M, Huang Z, Deng Q, Zhou M, Chen $\mathrm{Z}$ and Wang L: Burden of hypertension in China: A nationally representative survey of 174621 adults. Int J Cardiol 227: $516-523,2017$
22. Yang L, Yan J, Tang X, Xu X, Yu W and Wu H: Prevalence, awareness, treatment, control and risk factors associated with hypertension among adults in southern China, 2013. PLoS One 11: e0146181, 2016

23. Li D, Lv J, Liu F, Liu P, Yang X, Feng Y, Chen G and Hao M: Hypertension burden and control in mainland China: Analysis of nationwide data 2003-2012. Int J Cardiol 184: 637-644, 2015.

24. Lu J, Lu Y, Wang X, Li X, Linderman GC, Wu C, Cheng X, Mu L, Zhang H, Liu J, et al: Prevalence, awareness, treatment, and control of hypertension in China: Data from 1-7 million adults in a population-based screening study (China PEACE Million Persons Project). Lancet 390: 2549-2558, 2017.

25. Su M, Zhang Q, Bai X, Wu C, Li Y, Mossialos E, Mensah GA, Masoudi FA, Lu J, Li X, et al: Availability, cost, and prescription patterns of antihypertensive medications in primary health care in China: A nationwide cross-sectional survey. Lancet 390: 2559-2580, 2017.

26. NCD Risk Factor Collaboration (NCD-RisC): Worldwide trends in diabetes since 1980: A pooled analysis of 751 population-based studies with 4.4 million participants. Lancet 387: 1513-1530, 2016.

27. Xu Y, Wang L, He J, Bi Y, Li M, Wang T, Wang L, Jiang Y, Dai M, Lu J, et al: Prevalence and control of diabetes in Chinese adults. JAMA 310: 948-959, 2013.

28. Pan XR, Yang WY, Li GW and Liu J: Prevalence of diabetes and its risk factors in China, 1994. National diabetes prevention and control cooperative group. Diabetes Care 20:1664-1669, 1997.

29. Wang L, Gao P, Zhang M, Huang Z, Zhang D, Deng Q, Li Y, Zhao Z, Qin X, Jin D, et al: Prevalence and ethnic pattern of diabetes and prediabetes in China in 2013. JAMA 317: 2515-2523, 2017.

30. Chinese Diabetes Society: Chinese guideline for the prevention and treatment of type 2 diabetes mellitus (2017 edition). Chin J Diabetes Mellitus 10: 4-67, 2018 (In Chinese).

31. Chan JC, Zhang Y and Ning G: Diabetes in China: A societal solution for a personal challenge. Lancet Diabetes Endocrinol 2: 969-979, 2014

32. International Diabetes Federation: IDF diabetes atlas seventh edition. 2015. Available: http://www.diabetesatlas.org/ resources/2015-atlas.html.

33. Wu S, Huang Z, Yang X, Zhou Y, Wang A, Chen L, Zhao H, Ruan C, Wu Y, Xin A, et al: Prevalence of ideal cardiovascular health and its relationship with the 4-year cardiovascular events in a northern Chinese industrial city. Circ Cardiovasc Qual Outcomes 5: 487-493, 2012.

34. Whelton PK, Carey RM, Aronow WS, Casey DE Jr, Collins KJ, Dennison Himmelfarb C, DePalma SM, Gidding S, Jamerson KA, Jones DW, et al: 2017 ACC/AHA/AAPA/ABC/ $\mathrm{ACPM} / \mathrm{AGS} / \mathrm{APh} \mathrm{A} / \mathrm{ASH} / \mathrm{ASPC} / \mathrm{NMA} / \mathrm{PCNA}$ guideline for the prevention, detection, evaluation, and management of high blood pressure in adults: A report of the American College of Cardiology/American heart association task force on clinical practice guidelines. Hypertension 71: e13-e115, 2018.

35. O'Brien E and Fitzgerald D: The history of blood pressure measurement. J Hum Hypertens 8: 73-84, 1994.

36. James PA, Oparil S, Carter BL, Cushman WC, DennisonHimmelfarb C, Handler J, Lackland DT, LeFevre ML, MacKenzie TD, Ogedegbe O, et al: 2014 evidence-based guideline for the management of high blood pressure in adults: Report from the panel members appointed to the Eighth Joint National Committee (JNC 8). JAMA 311: 507-520, 2014.

37. Fox CS, Pencina MJ, Meigs JB, Vasan RS, Levitzky YS and D'Agostino RB Sr: Trends in the incidence of type 2 diabetes mellitus from the 1970s to the 1990s: The Framingham Heart Study. Circulation 113: 2914-2918, 2006.

38. Hosmer DH and Lemeshow S: Applied Logistic Regression. 2nd edition. Wiley\&Sons, New York, NY, pp67-82, 2000.

39. Maeda Y, Inoguchi T, Etoh E, Kodama Y, Sasaki S, Sonoda N, Nawata H, Shimabukuro M and Takayanagi R: Brachial-ankle pulse wave velocity predicts all-cause mortality and cardiovascular events in patients with diabetes: The Kyushu prevention study of atherosclerosis. Diabetes Care 37: 2383-2390, 2014.

40. Turin TC, Kita Y, Rumana N, Takashima N, Kadota A, Matsui K, Sugihara H, Morita Y, Nakamura Y, Miura K and Ueshima H: Brachial-ankle pulse wave velocity predicts all-cause mortality in the general population: Findings from the Takashima study, Japan. Hypertens Res 33: 922-925, 2010. 
41. Ninomiya T, Kojima I, Doi Y, Fukuhara M, Hirakawa Y, Hata J, Kitazono $\mathrm{T}$ and Kiyohara Y: Brachial-ankle pulse wave velocity predicts the development of cardiovascular disease in a general Japanese population: The Hisayama study. J Hypertens 31: 477-483, 2013

42. Song Y, Wu S, Liu X and Qi X: Association of systolic blood pressure levels with cardiovascular and cerebrovascular events and all-cause mortality: A result from the Kailuan study. Blood Press Monit 21: 149-154, 2016.

43. Walsh KB, Woo D, Sekar P, Osborne J, Moomaw CJ,Langefeld CD and Adeoye O: Untreated hypertension: A powerful risk factor for lobar and nonlobar intracerebral hemorrhage in whites, blacks, and hispanics. Circulation 134: 1444-1452, 2016.

44. van Rooy MJ and Pretorius E: Obesity, hypertension and hypercholesterolemia as risk factors for atherosclerosis leading to ischemic events. Curr Med Chem 21: 2121-2129, 2014.

45. Hollander W: Role of hypertension in atherosclerosis and cardiovascular disease. Am J Cardiol 38: 786-800, 1976.

46. Marino A, Zhang Y, Rubinelli L, Riemma MA, Ip JE and Di Lorenzo A: Pressure overload leads to coronary plaque formation, progression, and myocardial events in $\mathrm{ApoE}^{-/-}$mice. JCI Insight 4: pii: 128220, 2019.

47. Lv P, Jin H, Liu Y, Cui W, Peng Q, Liu R, Sun W, Fan C, Teng Y, Sun W and Huang Y: Comparison of risk factor between lacunar stroke and large artery atherosclerosis stroke: A cross-sectional study in China. PLoS One 11: e0149605, 2016.

48. Safar ME and London GM: Therapeutic studies and arterial stiffness in hypertension: recommendations of the European society of hypertension. The clinical committee of arterial structure and function. Working group on vascular structure and function of the European society of hypertension. J Hypertens 18: 1527-1535, 2000.
49. Xiao H, Butlin M, Tan I and Avolio A: Effects of cardiac timing and peripheral resistance on measurement of pulse wave velocity for assessment of arterial stiffness. Sci Rep 7: 5990, 2017.

50. Chen MY, Zhao CC, Li TT, Zhu Y, Yu TP, Bao YQ, Li LX and Jia WP: Serum uric acid levels are associated with obesity but not cardio-cerebrovascular events in Chinese inpatients with type 2 diabetes. Sci Rep 7: 40009, 2017.

51. Linderman GC, Lu J, Lu Y, Sun X, Xu W, Nasir K, Schulz W, Jiang L and Krumholz HM: Association of Body Mass index with blood pressure among 1.7 million Chinese adults. JAMA Netw Open 1: e181271, 2018.

52. Erem C, Hacihasanoglu A, Deger O, Kocak M and Topbas M: Prevalence of dyslipidemia and associated risk factors among Turkish adults: Trabzon lipid study. Endocrine 34: 36-51, 2008.

53. Fan D, Li L, Li Z, Zhang Y, Ma X, Wu L and Qin G: Effect of hyperlipidemia on the incidence of cardio-cerebrovascular events in patients with type 2 diabetes. Lipids Health Dis 17: 102, 2018.

54. Ohkuma T, Ninomiya T, Tomiyama H, Kario K, Hoshide S, Kita Y, Inoguchi T, Maeda Y, Kohara K, Tabara Y, et al: Brachial-ankle pulse wave velocity and the risk prediction of cardiovascular disease: An individual participant data meta-analysis. Hypertension 69: 1045-1052, 2017.

This work is licensed under a Creative Commons Attribution-NonCommercial-NoDerivatives 4.0 International (CC BY-NC-ND 4.0) License. 